\title{
STUDY OF RAPIDITY GAP EVENTS IN HADRONIC Z DECAYS WITH L3 DETECTOR
}

\author{
SWAGATO BANERJEE \\ for the L3 collaboration, CERN. \\ E-mail: Swagato.Banerjee@cern.ch
}

\begin{abstract}
Rapidity gaps have been studied in well separated completely symmetric 3 jet events from hadronic $\mathrm{Z}$ decays to search for colour singlet exchange. Asymmetries in particle flow and angular separation, defined with respect to the inter-jet regions, are found to be sensitive to the exchange of a colour singlet object instead of a colour octet gluon. From a comparison of the distributions observed in the L3 experiment and Monte Carlo predictions, a limit is obtained on the fraction of colour singlet exchange in data.
\end{abstract}

\section{Introduction}

\subsection{Colour Singlet Exchange}

Events with large rapidity gaps, attributed to colour singlet exchange (CSE), have been observed at Hera [1] and the Tevatron [2]. By crossing symmetry, we may expect similar gaps in three jet hadronic $\mathrm{Z}$ decays (figure 1). This

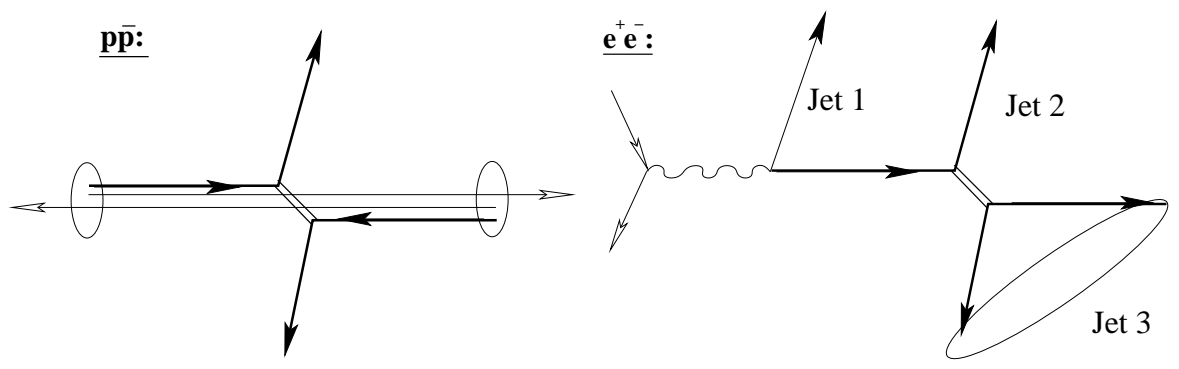

Figure 1. CsE in $\mathrm{p} \overline{\mathrm{p}}$ (left) and in $\mathrm{e}^{+} \mathrm{e}^{-}$(right) reactions shown by double lines.

study searches for such gaps by exploiting differences in colour flow in three jet topology between CsE and colour octet exchange (COE): in the latter colour flow is present between the $\mathrm{qg}$ and $\overline{\mathrm{q} g}$ gaps and is inhibited by destructive interference in the q⿳亠口冖 gap, while in the former case colour flow occurs only in the q⿳亠口冖 gap (figure 2). 

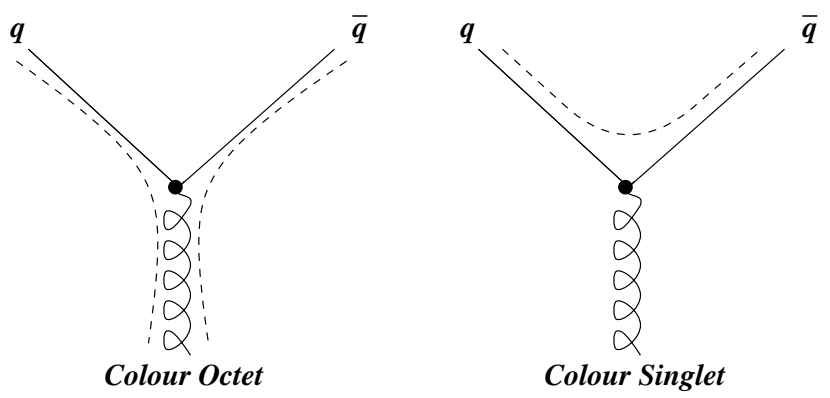

Figure 2. Colour flow in CoE (left) and CSE (right) shown by dotted lines.

\subsection{QCD models}

For this study, the JETSET Parton Shower program has been used to model Coe. Two toy models have been used to simulate the desired colour flow in CsE: events of type $\mathrm{q} \overline{\mathrm{q}} \gamma$ with a photon effective mass as in the gluon jet mass distribution are generated, and the photon is then replaced by a boosted two jet event (model CS0), or by a gluon fragmenting independently (model CS2). The RATHSman model, tuned to L3 hadronic Z decay data with string re-interaction probability set to 0.1 , is also studied [3].

\section{Methodology}

Three jet events are selected at fixed jet resolution parameter, set to 0.05 in the JADE algorithm (default) and to 0.01, 0.02 in the DURHAM algorithm (cross-check), with inter-jet angles within $\pm 30^{\circ}$ from the symmetric Mercedes topology. Particle momenta are projected onto the event plane defined by the two most energetic jets, and then all the angles (measured from most energetic jet) are rescaled so as to align jets at $0^{\circ}-120^{\circ}-240^{\circ}$ for uniformity in event-toevent comparison. To quantify the inter-jet gap angle, two definitions are used: the minimum opening angle of the particles measured from the bisector in each gap ( $B$-angle), and maximum separation angle between adjacent particles in each gap $(S$-angle). Ordering the jets such that jets 1, 2 are from primary quarks, and referring to $B(S)$-angle between jets $i, j$ generically as $\theta_{i j}(i, j=$ $1,3)$, gap asymmetries are defined as: $\mathrm{A}_{12}=\frac{-\theta_{12}+\theta_{23}+\theta_{31}}{\theta_{12}+\theta_{23}+\theta_{31}}$, and cyclically for the other gaps. Reduced colour flow and thereby larger separation for CsE in gaps 23, 31 (together referred to as qg) with respect to gap 12, should thus make $\mathrm{A}_{12}$ peak for positive values more strongly for CsE than for CoE.

cone-proc: submitted to World Scientific on December 25, 2018 


\section{Results}

The analysis is performed with 2 million hadronic $\mathrm{Z}$ decay events recorded by the L3 detector during 1994-95 corresponding to a luminosity of $75.1 \mathrm{pb}^{-1}$ 纤. For the three jets, labelled in order of decreasing energy, the probability of having a gluon in jet 3 in the default jet reconstruction is estimated using JETSET to be $69 \%$, reducing to $48 \%$ for Mercedes topology. In order to distinguish quark jets from gluon or colour singlet jets, quark jets are tagged by demanding that the b-tag discriminants of jets 1 and 2 are above a certain cut-off $\left(\mathrm{B}_{1}\right)$ and that of jet 3 is below a second cut-off $\left(\mathrm{B}_{2}\right)$. The selection, optimized to give the best sensitivity, tags 2668 events with $\mathrm{B}_{1}=1.25, \mathrm{~B}_{2}=$ 1.5 , corresponding to a probability of identifying a gluon in jet 3 as $78 \%$, the probability being varied by $10 \%$ for systematic studies. The data asymmetry distributions, corrected for detector effects as well as flavour composition, using detector simulated JETSET events with quarks jets from all flavours tagged in the first two jets, are compared to different models, all normalized to unit area, in figures 3 , 4. The systematic errors on bin-content from jet reconstruction $(\sim 4-7 \%)$ and tagging $(\sim 3-8 \%)$ have been added in quadrature.
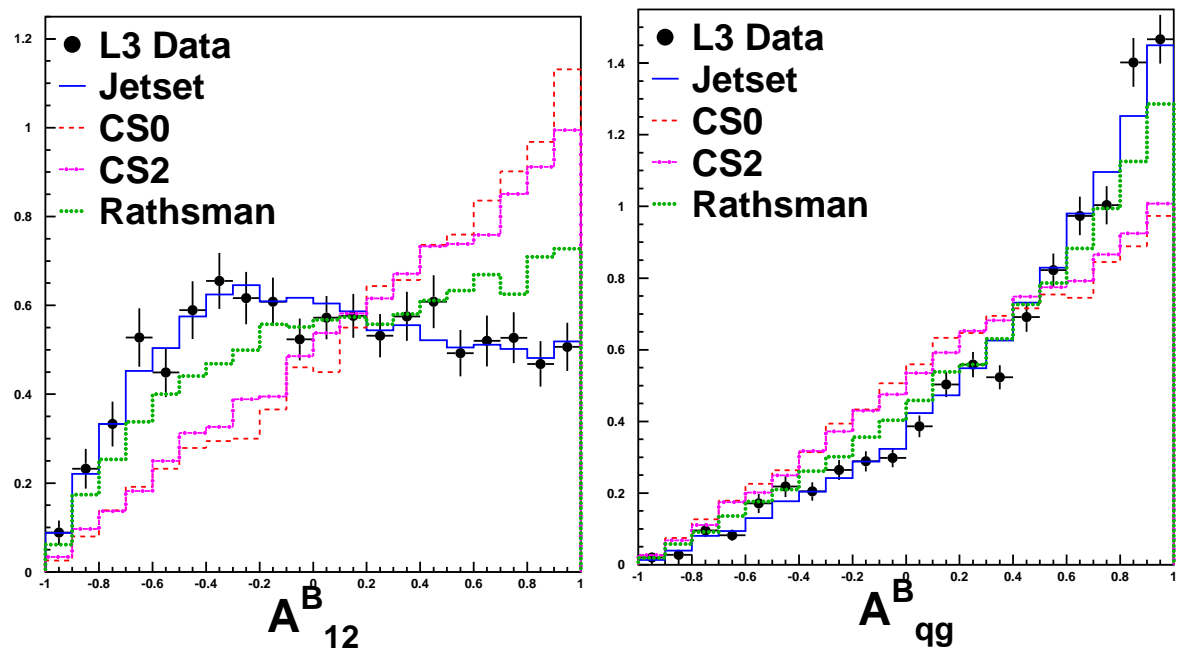

Figure 3. Minimum bisector angle gap asymmetries for gaps 12 and qg.

The data are consistent with COE as modelled by JETSET. The probability for having a $\chi^{2}$ greater than the observed value between data and RATHSMAN 
is $\mathcal{O}\left(10^{-6}\right)$. Fits to data with admixture of a fraction of CSE and remaining CoE are consistent with zero admixture. Combining the asymmetry between gaps 12 and qg, the upper limit of the fraction of CSE present in data is estimated to be between $7 \%$ to $9 \%$ at $95 \%$ confidence level.
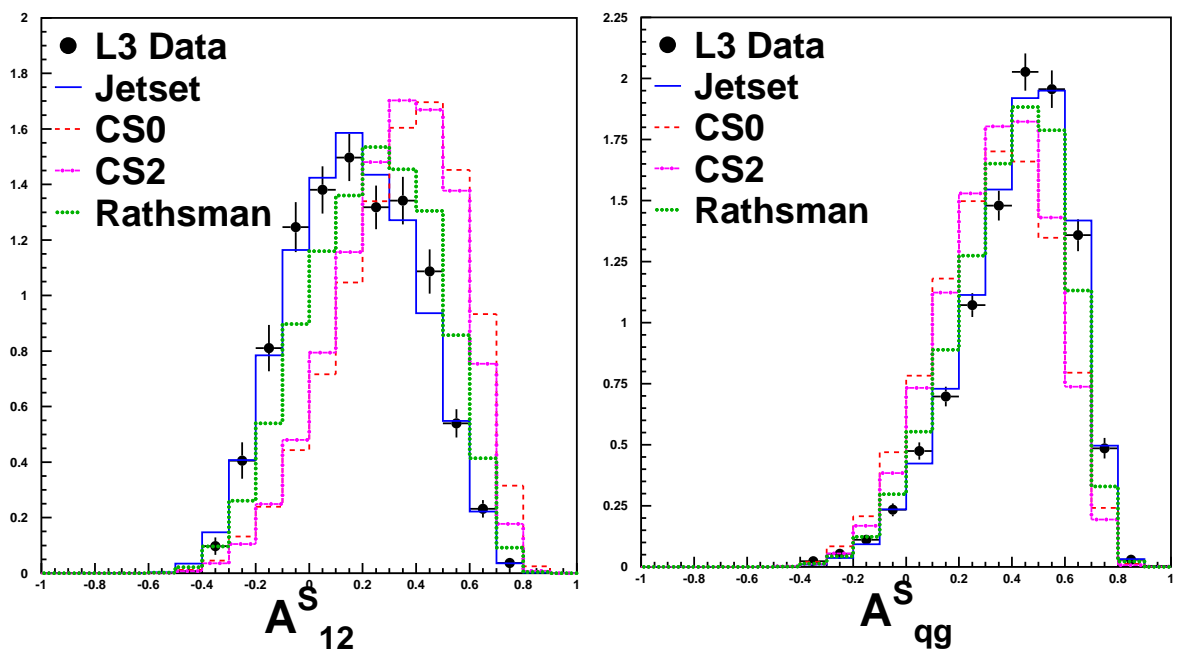

Figure 4. Maximum separation angle gap asymmetries for gaps 12 and qg.

\section{Acknowledgments}

I thank Sunanda Banerjee and John Field for their valuable collaborative efforts in this work.

\section{References}

1. H1 collaboration, T. Ahmed etal., Nucl. Phys. B429 (1994) 477; Zeus collaboration, M. Derrick etal., Phys. Lett. B369 (1996) 55.

2. DØ collaboration, B. Abbott etal., Phys. Lett. B440 (1998) 189; CDF collaboration, T. Affolder etal., Phys. Rev. Lett. 85 (2000) 4215.

3. J. Rathsman etal., Phys. Lett. B452 (1999) 364; Sunanda Banerjee and Swagato Banerjee, L3 Note \# 2715 (2001).

4. Contributions to the summer conferences '01, L3 note \#2684 (2001); Swagato Banerjee, Ph.D. Thesis, University of Mumbai (2000). 